Original Research Paper

\title{
Isolation and Screening of Wine Yeasts from Grapes in Yalu River Valley China for Fermentation Performance
}

\author{
${ }^{1,2}$ Xiaochun Yu, ${ }^{1,2}$ Jing Xu, ${ }^{1}$ Yuan Peng Li and ${ }^{1}$ Xian Peng Li \\ ${ }^{1}$ College of Food Science and Engineering, Tonghua Normal University, Tonghua 134002, China \\ ${ }^{2}$ Development Engineering Center of Edible Plant Resources of Changbai Mountain, Tonghua 134002, China
}

\author{
Article history \\ Received: 04-05-2021 \\ Revised: 26-07-2021 \\ Accepted: 03-08-2021 \\ Corresponding Author: \\ Jing Xu \\ College of Food Science and \\ Engineering, Tonghua Normal \\ University, Tonghua 134002, \\ China \\ E-mail: 122294352@qq.com
}

\begin{abstract}
In order to further explore the diversity of yeast in grape brewing in Yalu River Valley, northern China and to better exploit and utilize the yeast resources in a grape producing area, we investigated soil, grape residue after juice extraction and naturally fermented grape juice samples collected from grape plantations in the Yalu River Valley in this study. Twelve yeast strains were identified using colony and cell morphology and molecular biology. Three Saccharomyces cerevisiae strains from different sources, EN2, BZ1 and EZ2, were selected to determine their acid, Sodium dioxide $\left(\mathrm{SO}_{2}\right)$, glucose and ethanol tolerance. The results showed that EN2 exhibited significant acid and $\mathrm{SO}_{2}$ tolerance, while BZ1 and EZ2 exhibited significant glucose and ethanol tolerance. These three $S$. cerevisiae strains showed good growth and fermentation characteristics and could be used as development strains for brewing wine. Results of this study could be useful in breeding, wine yeast-strain research and wine product development.
\end{abstract}

Keywords: Wine, Yeast Strain, Acid Tolerance, Alcohol Tolerance, Ice Wine, Regional Characteristics

\section{Introduction}

Yeasts are important microorganisms in wine brewing that convert the sugar in grape juice to ethanol, carbon dioxide and other flavor-producing substances, such as fusels, aldehydes, acids and esters (Romano et al., 2008; Vernocchi et al., 2011). The yeast metabolites in wine fermentation significantly affect the flavor components, color and taste of wines; therefore, the types and characteristics of various yeast strains are important in wine production (Moreira et al., 2008; Ye et al., 2013; Lleixà et al., 2016). Choosing a suitable yeast with good fermentation performance for winemaking ensures the proper development of unique qualities and characteristics of grape raw materials. Current research primarily focuses on the screening and use of yeasts from various wine-producing areas (Heard, 1999; Nikolaou et al., 2006; Martínez et al., 2007). Compared with commercial active dry Saccharomyces cerevisiae, fermentation using local $S$. cerevisiae strains from grape-producing areas is more conducive to the formation and development of varieties and styles of regional wines and in overcoming the wine-homogenization phenomenon. In addition, studies have shown that non- $S$. cerevisiae participate in wine fermentation and can produce some aromatic components to enhance the aroma and positively affect the taste and quality of wine (Čuš and Jenko, 2013; Dashko et al., 2015; Maturano et al., 2015).

It is difficult for commercial yeasts to effectively represent the flavor of wine from different regions, especially ice wine, which can limit the production and development of wine from different regions to a certain extent. The Yalu River Valley, for example Beibinghong, is rich in raw grapes for ice wine brewing. Strict requirements are recommended for $S$. cerevisiae when brewing ice wine due to the high sugar and high acid within the raw materials and the low temperature environment during the fermentation process. Therefore, it is necessary to develop new. S cerevisiae according to the characteristics of the ice grape (Shen et al., 2020). The Yalu River Valley is located at $41^{\circ} \mathrm{N}$ latitude in northern China, the same latitude as the Rhone Valley in Bordeaux, France and the Napa Valley in California, United States; it is the "golden latitude" for grape cultivation in China (Liu et al., 2017). It is also an important location for ice wine. The superior geographical environment of the Yalu River Valley provides unique natural conditions for the growth of grapes and suitable conditions for screening and acquisition of high-quality yeast germplasm resources. In this study, soil, grape residue after juice extraction and naturally fermented grape juice samples were collected from grape plantations in the Yalu River Basin. A 
preliminary study was conducted on the fermentation performance of the isolated and identified yeast strains.

\section{Materials and Methods}

Materials

\section{Media and Chemicals}

Yeast extract Peptone Dextrose (YPD) containing 2\% glucose, $2 \%$ peptone, $1 \%$ yeast extract and $2 \%$ agar sterilized at $121^{\circ} \mathrm{C}$ for $20 \mathrm{~min}$ was used for the yeast screening and slant media (Nevoigt et al., 2000).

The yeast identification medium was Wallerstein Laboratory (WL) agar medium containing $0.5 \%$ yeast extract, $0.5 \%$ tryptone, $5 \%$ glucose, $2 \%$ agar, $0.055 \%$ potassium dihydrogen phosphate, $0.0425 \%$ potassium chloride, $0.0125 \%$ calcium chloride, $0.00025 \%$ ferric chloride, $0.0125 \%$ magnesium sulfate, $0.00025 \%$ manganese sulfate and $0.0022 \%$ bromocresol green, $\mathrm{pH}$ 6.5 sterilized at $121^{\circ} \mathrm{C}$ for $20 \mathrm{~min}$ (Di Maio et al., 2011).

The basic yeast tolerance medium contained $2 \%$ glucose, $2 \%$ peptone and $1 \%$ yeast extract sterilized at $121^{\circ} \mathrm{C}$ for $20 \mathrm{~min}$.

The agarose gel was obtained from Biowest Technology Co., Ltd. The Taq DNA polymerase and dNTP were purchased from TransGen Biotech Co., Ltd. The DNA extraction and agarose-gel DNA recovery kits were purchased from TianGen Biotech Co., Ltd. The standard molecular weight DNA marker DL-3000 was purchased from Solarbio Science and Technology Co., Ltd. The methylene blue, glucose, peptone, yeast extract and agar were analytically pure reagents purchased from Sinopharm Chemical Reagent Co., Ltd.

\section{Soil and Yeast Isolate Samples}

The soil samples were collected from the Beibinghong grape plantation in the Yalu River Valley in northern China. The grape residue samples after juicing and the naturally fermented grape juice samples were obtained from Beibinghong, Shuanghong and Gongniang No.1 grapes.

\section{Methods}

\section{Isolation and Screening of Yeast Strains from Plantation Soil Samples}

One-gram soil samples were aseptically weighed and placed in a flask with $100 \mathrm{~mL}$ sterile water, in a constant-temperature incubator (MJ-70-I, Yiheng Shanghai, China) and oscillated at $28^{\circ} \mathrm{C}$ for $30 \mathrm{~min}$. One milliliter of the mixture was added to $9 \mathrm{~mL}$ sterile water for a $10^{-7}$ gradient dilution, from which $0.1 \mathrm{~mL}$ aliquots of varying concentrations were added to YPD medium and cultivated at $28^{\circ} \mathrm{C}$ for $2-3$ days. Colonies with different morphologies were inoculated onto the media using the continuous streaking method and slant-stored at $4{ }^{\circ} \mathrm{C}$.

\section{Yeast Isolation from Grape Residue After Juicing}

After juicing, approximately one-gram samples of grape residue were aseptically weighed and the yeast isolates were used to inoculate the soil samples. Morphologically different colonies were inoculated onto media plates using the continuous streaking method, purified and slant-stored at $4^{\circ} \mathrm{C}$.

\section{Isolation with Yeast from Naturally Fermented Grape Juice}

One milliliter of grape naturally fermentation juice was added to $9 \mathrm{~mL}$ sterile water for gradient dilution to $10^{-7}$, from which. $0.1 \mathrm{~mL}$ aliquots of varying concentrations were added to YPD medium and cultivated at $28^{\circ} \mathrm{C}$ for $2-3$ days. Morphologically different colonies were inoculated onto media plates using the continuous streaking method, purified and slant-stored at $4{ }^{\circ} \mathrm{C}$.

\section{Yeast Strain Screening on WL Sedium}

The strains obtained from the preliminary screening were inoculated onto WL medium using the continuous streaking method and cultured at $28^{\circ} \mathrm{C}$ for 3-7 days. The colony color and morphology were observed and recorded.

\section{Microscopic Observation of Yeast Strains}

A light microscope (CX33, Olympus, Japan) was used to screen the yeast strains. A small amount of yeast was mixed with $0.1 \%$ methylene blue staining solution to stain the yeast cells; after 2-3 min of staining, the cell morphology was observed under microscope with $16 \times 40$ times.

\section{Molecular Biological Identification of Yeast}

The yeast strain genomic DNA was extracted as a template using a DNA extraction kit. The forward primer NL1 (5'-GCATATCAATAAGCGGAGGAAAAG-3') and reverse primer NL4 (5'-GGTCCGTGTTTCAAGACGG-3') were used to amplify the yeast $26 \mathrm{~S}$ rDNA D1/D2 region. The PCR-amplified fragments were sequenced by Shenggong Bioengineering Co., Ltd (Shanghai) and the sequences were compared with the GenBank database using BLAST software.

\section{Determination of S. cerevisiae Strain Fermentation Performance and Acid Tolerance}

Based on the results of strain identification, combined with the pretests and references, three $S$. cerevisiae strains, BZ1, EN2 and EZ2, from different sources were selected for fermentation performance tolerance testing (Pallmann et al., 2001; Zhang et al., 2016). The three strains were inoculated onto YPD liquid media with $\mathrm{pH}$ values of $2.0,2.5,3.0,3.5$ and 4.0 and $2 \%$ inoculum volume. The samples were incubated at $28^{\circ} \mathrm{C}$ for $24 \mathrm{~h}$ and 
the absorbance value was measured at $600 \mathrm{~nm}$ wavelength using a spectrophotometer (7200, Unico, China).

\section{Determination of $S$. cerevisiae Sodium Dioxide Tolerance}

Sodium bisulfite $\left(\mathrm{NaHSO}_{3}\right)$ was added to five YPD liquid media to bring the Sodium dioxide $\left(\mathrm{SO}_{2}\right)$ concentrations to $100,150,200,250$ and $300 \mathrm{mg} / \mathrm{L}$. The activated yeast strains BZ1, EN2 and EZ2, were incubated with a $2 \%$ inoculum, cultured at $28^{\circ} \mathrm{C}$ for $24 \mathrm{~h}$ and the absorbance was determined.

\section{Determination of $S$. cerevisiae Strain Sugar Tolerance}

YPD liquid media containing 10, 15, 20, 25, 30 and $35 \%$ glucose were prepared. The activated strains BZ1, EN2 and EZ2, were inoculated with a $2 \%$ inoculum. The absorbance was measured after culturing for $24 \mathrm{~h}$.

\section{Determination of S. cerevisiae Alcohol Tolerance}

YPD liquid media with ethanol concentrations of 10, 12, 14, 16 and $18 \%(\mathrm{v} / \mathrm{v})$ were prepared. The activated strains BZ1, EN2 and EZ2, were inoculated with a $2 \%$ inoculum volume. The samples were incubated at $28^{\circ} \mathrm{C}$ for $24 \mathrm{~h}$ and the absorbance value was measured.

\section{Results}

\section{Yeast Strain Screening}

In this study, 23 yeast strains were isolated from various Yalu River Valley grape plantation samples and screened. Four strains were isolated from soil samples, nine from the grape residue after juice extraction and ten strains from naturally fermented grape juice. The sources and strain numbers of the isolated strains are listed in Table 1.

\section{Identification of Yeast Strains on WL Medium}

The 23 yeast strains were inoculated onto WL medium and cultured at $28^{\circ} \mathrm{C}$ for 4 days, of which 12 conformed to the colony morphology of yeast on WL medium. The morphological characteristics of 12 strains are shown in Fig. 1. One strain was isolated from a soil sample, six from the grape residue after juice extraction and five from naturally fermented grape juice. The yeast strain number, sample source and basic strain morphology are shown in Table 2 .

\section{Microscopic Observation of Yeast}

The 12 yeast strains were examined under a microscope and identified by the morphological characteristics of their WL-medium colonies. The yeast cells were approximately spherical or oval-shaped, without pseudohyphae or spores. The morphology showed single, opposite, or group, single-terminal or double terminal budding. The microscopic morphology of the strains was consistent with yeast morphological characteristics (Garde-Cerdán et al., 2007).

\section{$26 S$ rDNA Identification of Yeast Strains}

The D1/D2 region of the yeast strain 26S rDNA was amplified by PCR and the homology of the gene sequences of each strain was compared with the GenBank database. The results showed that among the 12 strains, there were $10 \mathrm{~S}$. cerevisiae, 1 Meyerozyma guilliermondii and 1 Pichia membranifaciens. The homology was 100 or $99 \%$. The results of the $26 \mathrm{~S}$ rDNA yeast strain identification are shown in Table 3.

\section{S. cerevisiae Strain Acid Tolerance}

The acid tolerance of three $S$. cerevisiae strains, BZ1, EN2 and EZ2, was measured by inoculation onto YPD media with various $\mathrm{pH}$ levels. The results presented in Fig. 2 show that the growth of EN2 at $\mathrm{pH} 2.5$ was close to that at $\mathrm{pH} 3$ and slightly lower than at $\mathrm{pH} 4$, while that of BZ1 and EZ2 were significantly affected by low $\mathrm{pH}$. The results showed that EN2 exhibited significant acid resistance.

\section{S. cerevisiae $\mathrm{SO}_{2}$ Tolerance}

The results of the $\mathrm{SO}_{2}$ tolerance test on the three strains are shown in Fig. 3. It is necessary to add an appropriate amount of $\mathrm{SO}_{2}$ during wine production to inhibit harmful microorganisms, antioxidant activity and color changes (Maragatham and Panneerselvam, 2011). The ideal wine-making yeast ferments normally at a specific $\mathrm{SO}_{2}$ concentration. Fig. 3 shows that when the media $\mathrm{SO}_{2}$ concentration was $250 \mathrm{mg} / \mathrm{L}$, EN2 growth was normal, while BZ1 and EZ2 decreased, indicating that EN2 possessed significant $\mathrm{SO}_{2}$ tolerance.

\section{S. cerevisiae Sugar Tolerance}

The glucose tolerance of the various $S$. cerevisiae strains is shown in Fig. 4. Increased media glucose concentration inhibited EN2 and EZ2 growth and at $30 \%$, their growth was significantly inhibited, while that of BZ1 decreased slightly.

\section{S. cerevisiae Alcohol Tolerance}

The results of the alcohol-tolerance tests on three $S$. cerevisiae strains are shown in Fig. 5. The growth of EN2 and BZ1 was significantly affected by increased media alcohol content, while EZ2 maintained normal growth at $16 \%$ alcohol concentration. 


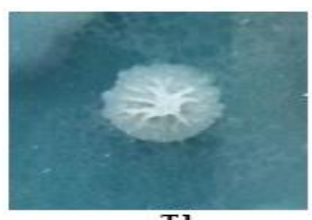

T1

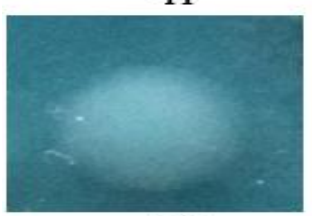

SN3

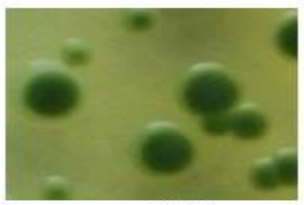

BZ2

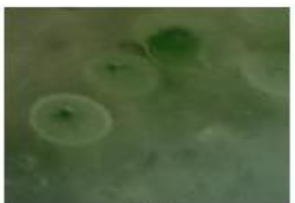

BN1

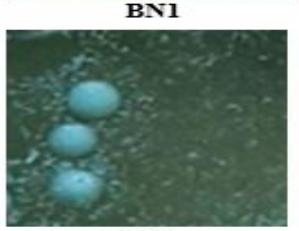

EN1

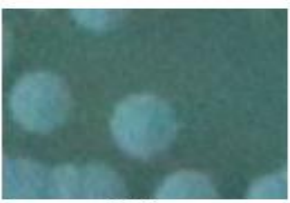

SZ1

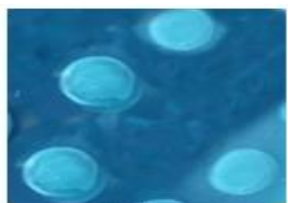

SN1

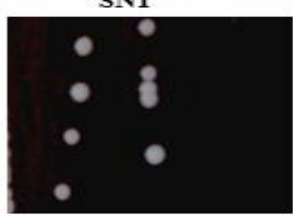

EN2

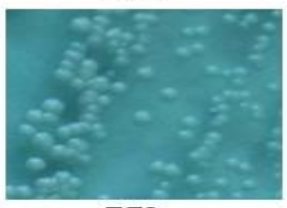

EZ1

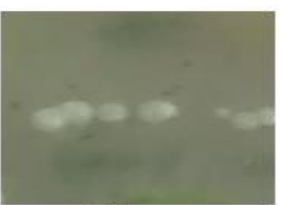

SN2

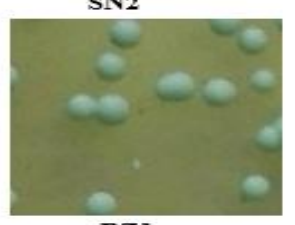

BZ1

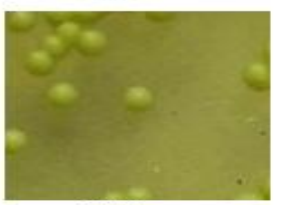

EZ2

Fig. 1: Morphology of yeasts from various sources strain T1 was obtained from plantation soil samples and was not S. cerevisiae. Strains BN1, SN1, SN2, SN3, EN1 and EN2 were obtained from the residue after juicing samples of Beibinghong, Shuanghong and Gongniang No.1, respectively. Strains BZ1, BZ2, SZ1, EZ1 and EZ2 were obtained from the natural fermented grape juice samples of Beibinghong, Shuanghong and Gongniang No.1, respectively and SZ1 was not S. cerevisiae

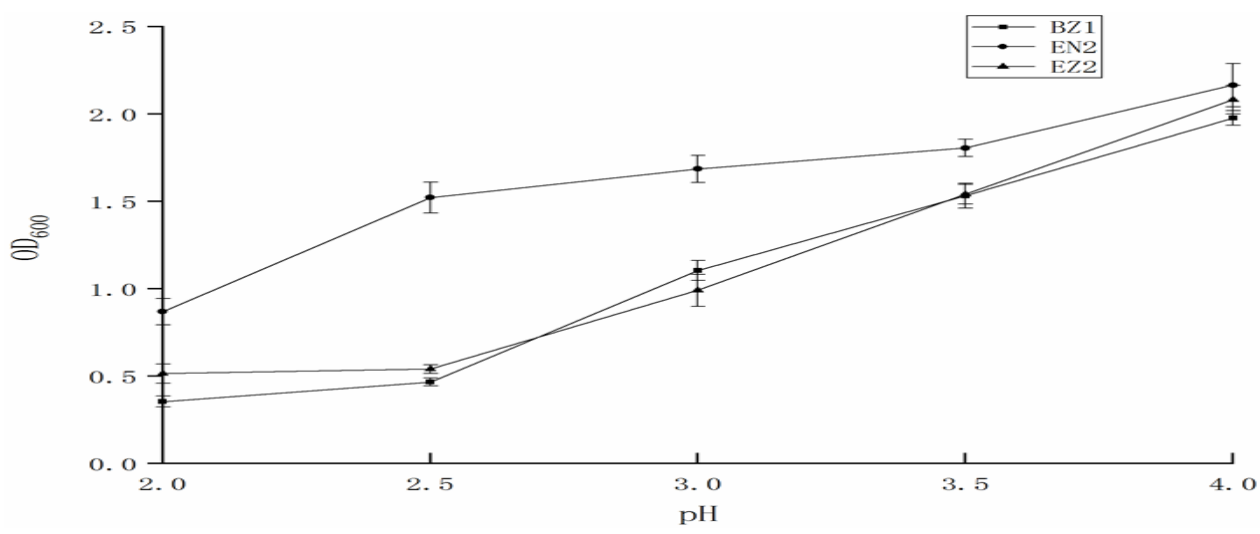

Fig. 2: S. cerevisiae strain acid tolerance the growth status of the three strains was different under variable acidity conditions and the strain EN2 could maintain good growth status under higher acidity conditions

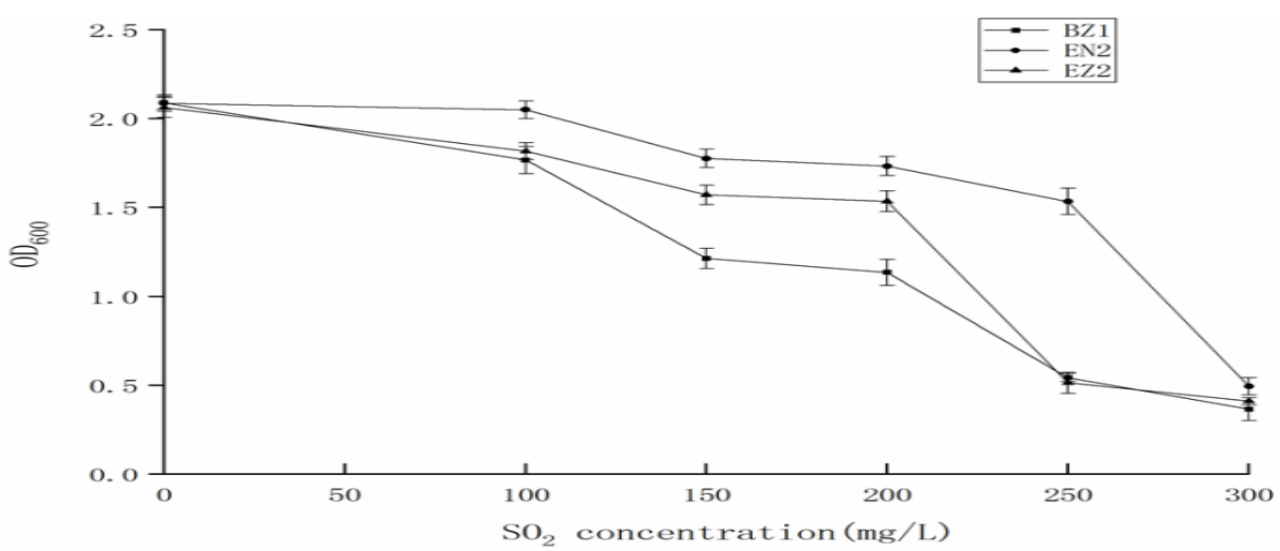

Fig. 3: $S$. cerevisiae strain sodium dioxide tolerance during the process of wine brewing, the addition of $\mathrm{SO}_{2}$ affects the growth of yeast. When the addition of $\mathrm{SO}_{2}$ was $250 \mathrm{mg} / \mathrm{L}$, the growth of strain $\mathrm{EN} 2$ was good. When the addition of $\mathrm{SO}_{2} \mathrm{was} 300 \mathrm{mg} / \mathrm{L}$, the three yeast strains could not grow normally 


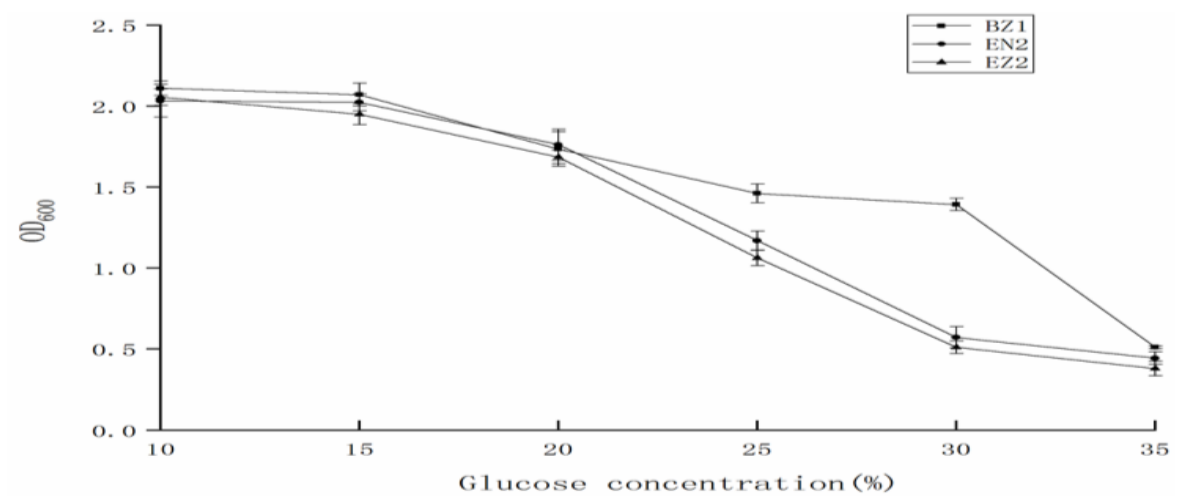

Fig. 4: $S$. cerevisiae strain glucose tolerance a high concentration of sugar can inhibit the growth of $S$. cerevisiae and affect the fermentation process. When the concentration of glucose reached 30\%, the growth of strain EN2 and EZ2 was inhibited, while strain BZ1 screened from Beibinghong juice maintained normal growth

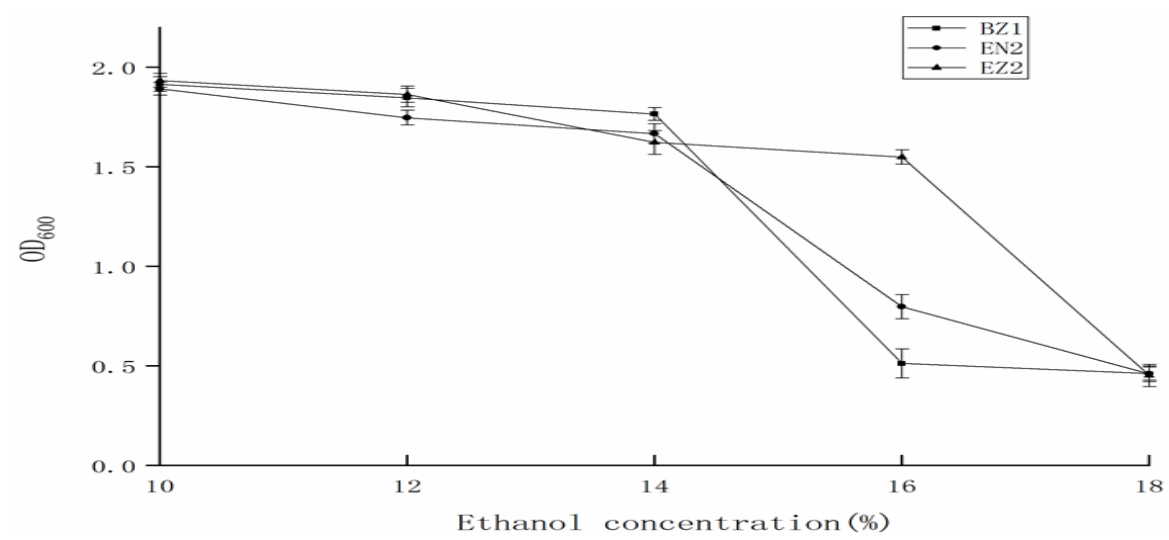

Fig. 5: $S$. cerevisiae strain alcohol tolerance the tolerance of $S$. cerevisiae to alcohol directly affects its fermentation ability. Strains BZ1, EN2 and EZ2 could grow in the medium with $14 \%$ alcohol concentration. Strain EZ2 could still maintain growth when the alcohol concentration was $16 \%$

Table 1: Yeast strains isolated from various sources

\begin{tabular}{lll}
\hline Sample Source & & \\
\hline--------------------------------------------- & Sample type & Strain number \\
\hline- & Soil & T1, T2, TH1, TX2 \\
Beibinghong & Grape residue after juice extraction & BN1, BN2 \\
Shuanghong & Grape residue after juice extraction & SN1, SN2, SN3, SN4, \\
Gongniang No.1 & Grape residue after juice extraction & EN1, EN2, EN3 \\
Beibinghong & Naturally fermented grape juice & BZ1, BZ2, BZ3, BZ4 \\
Shuanghong & Naturally fermented grape juice & SZ1, SZ2, SZ3 \\
Gongniang No.1 & Naturally fermented grape juice & EZ1, EZ2, EZ3 \\
\hline
\end{tabular}

Table 2: Colony morphology of yeast strains isolated from different sources

\begin{tabular}{lll}
\hline Strain number & Sample source & Strain morphology \\
\hline T1 & soil & Milky white, convex at center, dry, irregular edges \\
BN1 & Beibinghong grape residue after juice extraction & Dark green, convex in center, neat edges \\
SN1 & Shuanghong grape residue after juice extraction & White, creamy, neat edges \\
SN2 & Shuanghong grape residue after juice extraction & White, creamy, raised surface, neat edges \\
SN3 & Shuanghong grape residue after juice extraction & White, dry, irregular edges \\
EN1 & Gongning No.1 grape residue after juice extraction & Milky white, creamy, with raised surface and neat edges \\
EN2 & Gongning No.1 grape residue after juice extraction & White with neat edges \\
BZ1 & Beibinghong grape naturally fermented juice & White, raised surface, creamy, neat edges \\
BZ2 & Beibinghong grape naturally fermented juice & Dark green, raised surface, creamy, neat edges \\
SZ1 & Shuanghong grape naturally fermented juice & White, the surface is annular convex, dry, the edge is powdery \\
EZ1 & Gongniang No.1 grape naturally fermented juice & White, spherical raised, neat edges, creamy \\
EZ2 & Gongniang No.1 gape naturally fermented juice & Yellow-green, spherical convex, neat edges, creamy
\end{tabular}


Table 3: 26S rDNA identification of yeast strains

\begin{tabular}{llr}
\hline Strain number & Identification & Genebank serial number \\
\hline T1 & Meyerozyma guilliermondii & KX791362.1 \\
BN1 & Saccharomyces cerevisiae & MN648829.1 \\
SN1 & Saccharomyces cerevisiae & KY273294.1 \\
SN2 & Saccharomyces cerevisiae & LC496585.1 \\
SN3 & Saccharomyces cerevisiae & MT322857.1 \\
EN1 & Saccharomyces cerevisiae & MG641152.1 \\
EN2 & Saccharomyces cerevisiae & MH472657.1 \\
BZ1 & Saccharomyces cerevisiae & MT420738.1 \\
BZ2 & Saccharomyces cerevisiae & CP046092.1 \\
SZ1 & Pichia membranifaciens & MW009112.1 \\
EZ1 & Saccharomyces cerevisiae & CP046463.1 \\
EZ2 & Saccharomyces cerevisiae & MG017580.1
\end{tabular}

\section{Discussion}

WL medium can be used to identify and differentiate between yeasts, primarily based on colony color and morphology. It has been reported that the general morphological characteristics of $S$. cerevisiae on WL medium are milky white to green, with spherical protuberances, smooth surface, opaque and creamy consistency (Nielsen and Arneborg, 2007). In this study, 12 yeast strains from various sources were screened and identified by WL medium colony observation,

Culture conditions had a great influence on the growth of yeast. For example, the $\mathrm{pH}$ value can change the ionization degree of media nutrients, affecting cellular nutrient absorption and ultimately affecting growth (Xue et al., 2007).

Sugar is the substrate for alcohol production via fermentation and is the energy source for yeast; however, high sugar concentration can inhibit yeast growth, resulting in glucose repression and inhibition. Moreover, the high osmotic pressure at increased sugar concentrations leads to water loss from yeast cells and decreases their activity (Xu et al., 2014). Several metabolic systems, including transmission pathways and molecular response mechanisms, regulate yeast osmotic pressure under high sugar stress (Jin et al., 2020). The Beibinghong grape, developed in 2008, is a mountain hybrid with high cold resistance and yield. It is primarily cultivated in northeast China and is the most important ice-wine brewing mountain grape variety (Biasi et al., 2014). Generally, the sugar content of grape juice used for winemaking is about $250 \mathrm{~g} / \mathrm{L}$. The ice-wine production environment requires yeast that can tolerate high sugar and acid concentrations and low temperature (GuoHuan et al., 2017). The optimal alcohol content in industrial wine brewing is $10-13 \%$; higher alcohol concentrations can inhibit yeast growth. EZ2 selected from naturally fermented Gongniang No.1 grape juice exhibited significant alcohol tolerance up to $16 \%$.

\section{Conclusion}

Yeast is the most important strain in the brewing industry. It is the soul of wine quality and has a big influence on the color, aroma and flavor of a wine. In this study, 12 yeast strains from various sources were screened and identified by WL medium colony observation, microscopic observation and 26S rDNA sequencing. The results of the fermentation test showed that EN2 exhibited significant acid and $\mathrm{SO}_{2}$ tolerance and can tolerate certain acidity and $\mathrm{SO}_{2}$ at the concentration of $250 \mathrm{mg} / \mathrm{L}$. Strain BZ1 showed significant sugar tolerance, which can be fermented at a glucose concentration of $300 \mathrm{~g} / \mathrm{L}$. The results demonstrated that strain EZ2 had good alcohol tolerance and could still ferment at $16 \%$ alcohol volume fraction. The three strains have excellent fermentation performance and could be used as development strains for wine making. Further studies are required to investigate the wine-brewing performance of the yeasts to select strains that exhibit characteristic qualities of the grape-producing areas with better fermentation abilities.

\section{Acknowledgment}

Financial support: This study was funded by the Science and Technology Department of Jilin Province Key projects (Project number: 2018021045NY) and the Tonghua Normal University Scientific Research Project (Project number: 201841).

\section{Author's Contributions}

Xiaochun Yu: Participated in the experimental procedure and was responsible for data analysis and manuscript preparation.

Jing Xu: Participated in the experimental design and contributed to the writing and revision of the manuscript.

Yuan Peng Li: Contributed to the collection of samples, screening and identification of strains.

Xian Peng Li: Contributed to the fermentation performance and tolerance experiments and participated in data analysis. 


\section{Ethics}

All authors read and approved the final version and are responsible for any ethical issue that may arise after the publication of this manuscript.

\section{References}

Biasi, F., Deiana, M., Guina, T., Gamba, P., Leonarduzzi, G., \& Poli, G. (2014). Wine consumption and intestinal redox homeostasis. Redox Biology, 2, 795-802. doi.org/10.1016/j.bcp.2013.03.024

Čuš, F., \& Jenko, M. (2013). The influence of yeast strains on the composition and sensory quality of Gewürztraminer wine. Food Technology and Biotechnology, 51(4), 547-553.

https://hrcak.srce.hr/index.php?show=clanak\&id_cla nak_jezik=169441

Dashko, S., Zhou, N., Tinta, T., Sivilotti, P., Lemut, M. S., Trost, K., ... \& Piskur, J. (2015). Use of non-conventional yeast improves the wine aroma profile of Ribolla Gialla. Journal of Industrial Microbiology and Biotechnology, 42(7), 997-1010. doi.org/10.1007/s10295-015-1620-y

Di Maio, S., Polizzotto, G., Planeta, D., \& Oliva, D. (2011). A method to discriminate between the Candida stellata and Saccharomyces cerevisiae in mixed fermentation on WLD and lysine agar media. South African Journal of Enology and Viticulture, 32(1), 35-41. doi.org/10.21548/32-1-1364

Garde-Cerdán, T., Marsellés-Fontanet, A. R., AriasGil, M., Martín-Belloso, O., \& Ancín-Azpilicueta, C. (2007). Influence of $\mathrm{SO}_{2}$ on the consumption of nitrogen compounds through alcoholic fermentation of must sterilized by pulsed electric fields. Food Chemistry, 103(3), 771-777. doi.org/10.1016/j.foodchem.2006.09.018

GuoHuan, N., WenJun, S., YaBo, H., \& TingGang, W. (2017). Study on the performance of natural yeast in the ice wine Beibinghong of the Yalu River basin. Food Research and Development, 38(12), 162-166. https://www.cabdirect.org/globalhealth/abstract/2 0173271293

Jin, Y. N., Shu, N., Xie, S. Y., Zhang, B. X., Xiao, J. M., \& Lu, W. P. (2020). Effect of delayed harvest on volatile components in Beibinghong grape and distilled wine. Chn. Brw., 39(12), 140-145. doi.org/10.11882/j.issn.0254-5071.2020.12.027

Liu, H., Wenbing, H. E., Qiao, L. I., \& Zhang, L. (2017). Comparison of quality traits of four major grape cultivars planted in Tonghua. Food Sci., 38(17), 107-113. doi.org/10.7506/spkx1002-6630-201717018
Lleixà, J., Martin, V., Portillo, C., Carrau, F., Beltran, G., \& Mas, A. (2016). Comparison of the performances of Hanseniaspora vineae and Saccharomyces cerevisiae during winemaking. Front. Microbiol, 7, 338. doi.org/10.3389/fmicb.2016.00338

Maragatham, C., \& Panneerselvam, A. (2011). Isolation, identification and characterization of wine yeast from rotten papaya fruits for wine production. Advances in Applied Science Research, 2(2), 93-98. https://www.cabdirect.org/cabdirect/abstract/201133 46981

Martínez, C., Cosgaya, P., Vásquez, C., Gac, S., \& Ganga, A. (2007). High degree of correlation between molecular polymorphism and geographic origin of wine yeast strains. Journal of applied microbiology, 103(6), 2185-2195. doi.org/10.1111/j.1365-2672.2007.03493.x

Maturano, Y. P., Assof, M., Fabani, M. P., Nally, M. C., Jofré, V., Assaf, L. A. R., ... \& Vazquez, F. (2015). Enzymatic activities produced by mixed Saccharomyces and non-Saccharomyces cultures: relationship with wine volatile composition. Antonie Van Leeuwenhoek, 108(5), 1239-1256. doi.org/10.1007/s10482-015-0578-0

Moreira, N., Mendes, F., de Pinho, P. G., Hogg, T., \& Vasconcelos, I. (2008). Heavy sulphur compounds, higher alcohols and esters production profile of Hanseniaspora uvarum and Hanseniaspora guilliermondii grown as pure and mixed cultures in grape must. International journal of food microbiology, 124(3), 231-238.

doi.org/10.1016/j.ijfoodmicro.2008.03.025

Heard, G. (1999). Novel yeasts in winemaking: looking to the future. Food Australia, 51(8), 347-352.

https://pascal-

francis.inist.fr/vibad/index.php?action=getRecordDe tail\&idt=1907546

Nevoigt, E., Fassbender, A., \& Stahl, U. (2000). Cells of the yeast Saccharomyces cerevisiae are transformable by DNA under non-artificial conditions. Yeast, 16(12), 1107-1110. doi.org/10.1002/10970061(20000915)16:12<1107::AIDYEA608>3.0.CO;2-3

Nielsen, M. K., \& Arneborg, N. (2007). The effect of citric acid and $\mathrm{pH}$ on growth and metabolism of anaerobic Saccharomyces cerevisiae and Zygosaccharomyces bailii cultures. Food Microbiology, 24(1), 101-105. doi.org/10.1016/j.fm.2006.03.005

Nikolaou, E., Soufleros, E. H., Bouloumpasi, E., \& Tzanetakis, N. (2006). Selection of indigenous Saccharomyces cerevisiae strains according to their oenological characteristics and vinification results. Food Microbiology, 23(2), 205-211. doi.org/10.1016/j.fm.2005.03.004 
Pallmann, C. L., Brown, J. A., Olineka, T. L., Cocolin, L., Mills, D. A., \& Bisson, L. F. (2001). Use of WL medium to profile native flora fermentations. American Journal of Enology and Viticulture, 52(3), 198-203. https://www.ajevonline.org/content/52/3/198.short

Romano, P., Capece, A., Serafino, V., Romaniello, R., \& Poeta, C. (2008). Biodiversity of wild strains of Saccharomyces cerevisiae as tool to complement and optimize wine quality. World Journal of Microbiology and Biotechnology, 24(9), 1797-1802. doi.org/10.1007/s11274-008-9672-Z

Shen, J. Y., Zhang, B. Q., Duan, C. Q., \& Yan, G. L. (2020). Selection of indigenous Saccharomyces cerevisiae strains from spontaneous fermentation of Vidal icewine in Huanren region and evaluation of their oenological properties. Food Science, 41(2), 148-157. doi.org/10.7506/spkx1002-6630-20180903-022

Vernocchi, P., Ndagijimana, M., Serrazanetti, D. I., López, C. C., Fabiani, A., Gardini, F., ... \& Lanciotti, R. (2011). Use of Saccharomyces cerevisiae strains endowed with $\beta$-glucosidase activity for the production of Sangiovese wine. World Journal of Microbiology and Biotechnology, 27(6), 1423-1433. doi.org/10.1007/s11274-010-0594-1
Xu, X. L., Feng, G. L., Liu, H. W., Li, X. F., Zhao, G. L., \& Xiao, X. L. (2014). Isolation, identification and control of osmophilic spoilage yeasts in sweetened condensed milk. African Journal of Microbiology Research, 8(10), 1032-1039. doi.org/10.5897/AJMR2013.6203

Xue, J. X., XU, Y. W., YANG, Y., \& LIU, Y. L. (2007). Investigation on the Tolerance of Wild Saccharomyces cerevisiae. LIQUOR MAKING SCIENCE AND TECHNOLOGY, 6(156), 45. https://en.cnki.com.cn/Article_en/CJFDTotalNJKJ200706011.htm

Ye, M., Yue, T., Yuan, Y., \& Wang, L. (2013). Production of yeast hybrids for improvement of cider by protoplast electrofusion. Biochemical Engineering Journal, 81, 162-169. doi.org/10.1016/j.bej.2013.10.016

Zhang, J. J., Yang, X., Guo, C., Li, M. Y., Zhang W., \& Liu, C. (2016). Separation \& identification of yeasts from Pericarp of different Merlot grape strains and analysis of their difference. Liq. Mak. Science Technology, 2016(10), 22-26. doi.org/10.13746/j.njkj.2016212 